\title{
La société civile, de l'alerte à la controverse médiatisée
}

Civil society, from alarm to media controversy

\section{Céline Pascual Espuny}

\section{(2) OpenEdition}

\section{Journals}

\section{Édition électronique}

URL : http://journals.openedition.org/communicationorganisation/4531

DOI : 10.4000/communicationorganisation.4531

ISSN : $1775-3546$

\section{Éditeur}

Presses universitaires de Bordeaux

\section{Édition imprimée}

Date de publication : 1 juin 2014

Pagination : 115-126

ISBN : 978-2-86781-904-9

ISSN : $1168-5549$

Référence électronique

Céline Pascual Espuny, "La société civile, de l'alerte à la controverse médiatisée », Communication et organisation [En ligne], 45 | 2014, mis en ligne le 01 juin 2017, consulté le 10 décembre 2020. URL: http://journals.openedition.org/communicationorganisation/4531; DOI : https://doi.org/10.4000/ communicationorganisation.4531 


\title{
La société civile, de l'alerte à la controverse médiatisée
}

\author{
Céline Pascual Espunỵ'
}

Les dernières crises concernant la santé environnementale et les questions sanitaires, aujourd'hui sous les projecteurs médiatiques (Bisphénol A, éthers de glycol, aspartame, dioxine, etc.), montrent un nouveau visage de la société civile qui, non seulement s'approprie les sujets les plus techniques et spécialisés, mais devient lanceur d'alerte.

Quel est ce nouveau visage de la société civile ? Quel nouvel espace public se dessine?

Le lanceur d'alerte est désormais polymorphe, et c'est en soi une nouveauté que nous prenons comme constat initial de notre analyse : citoyens ou experts, professionnels, amateurs..., les procès se multiplient à l'initiative des lanceurs d'alerte et montrent un nouveau visage de ces nouveaux gardiens (Bournois, Bourion, 2008)

Parallèlement, les médias semblent être plus volontiers à l'écoute des lanceurs d'alerte ; l'accès aux médias de masse se précise, notamment par le biais d'enquêtes et de documentaires à charge. Le schéma communicationnel ainsi engendré se complexifie.

Enfin, le recours au droit environnemental, la multiplication des procès et de la jurisprudence qui en est issue, dessinent un espace public où la controverse devient l'un des moyens de faire bouger les lignes de rupture et les lignes de consensus sur des sujets devenus publics et d'intérêt général.

Cet article propose une relecture communicationnelle des analyses déjà existantes en sciences humaines et sociales sur l'alerte et les lanceurs d'alerte. L'angle choisi est nouveau : nous prenons l'alerte comme un phénomène communicationnel que nous suivons, de sa création, de son émetteur, jusqu'à sa finalité, son aboutissement dans l'espace public. Analyser les canaux et les transformations suivis par l'alerte ouvre un deuxième questionnement

1 Céline Pascual Espuny est maître de conférences à Aix-Marseille Université. Chercheur à l'IRSIC (Institut de Recherche en Sciences de l'Information et de la Communication), elle collabore également avec le Groupe Sup de Co Montpellier Business School Research Center ; celine.pascual@univ-amu.fr

Céline Pascual Espuny is senior lecturer at Aix-Marseille University. Researcher IRSIC (Research Institute of Information Science and Communication), she also works with the Groupe Sup de Co Montpellier Business School ResearchCenter. ; celine.pascual@univ-amu.fr 
parallèle, celui de ce nouveau visage de la société civile qui s'exprime dans l'alerte, qui se mobilise pour la transmission de celle-ci, qui s’investit dans sa diffusion, dans son interprétation et dans sa relecture.

Pour cet article exploratoire, nous mobilisons les notions centrales d'expertise (Keck, 2009), de controverse (Lascoumes, 2002, Callon et al, 2001), de rumeur (Morin, 1999, Boltanski et al, 2007), d'évènement (Quéré, 2005, Lemieux, 2000, Champagne, 1984) et d'espace public (Habermas, 1992, Schudson, 1995). Nous élargissons ensuite notre réflexion aux notions de crise et de risque (Beck, 1992).

Dans la première partie, en nous appuyant sur la littérature existante sur le lanceur d'alerte (Appelbaum, Grewal, Mousseau, 2006, Fisher et al, 1999, Martens, Kelleher, 2004, Miceli, 1985, 1996), nous nous concentrons sur la genèse de l'alerte, son contexte d'énonciation et nous analysons en particulier le statut et le profil de son émetteur, le lanceur d'alerte ainsi que la nouvelle logique médiatique qu'il suit.

Dans la deuxième partie, nous suivons l'alerte au travers de son passage dans les médias puis dans l'arène publique. Notre analyse permet d'envisager l'alerte comme message, comme rupture de sens, mais aussi comme évènement. L'alerte, envisagée comme phénomène communicationnel, nous invite à une relecture des analyses principalement issues de la sociologie et de la science politique. Nous nous concentrons en particulier sur la dynamique des controverses (Boltanski et al, 2007, Callon, 1981, Quéré, 2005) et nous ouvrons le champ en y croisant la question de l'expertise et des experts au regard de la théorie du leader d'opinion.

Enfin, dans la troisième partie, nous élargissons nos commentaires sur les concepts de crise et de risque, en tenant compte de la portée et de l'efficacité des alertes que nous avons mises en évidence dans nos analyses précédentes.

\section{L'alerte dans sa genèse : le poids de l'individu contre la norme}

Dans sa genèse, l'alerte est très fortement connotée par son lanceur et son contexte d'énonciation. Très vite, les premières recherches se sont portées sur la personnalité des lanceurs d'alerte, puis sur son impact sur l'organisation. L'alerte a évolué avec son temps, et la protection juridique des lanceurs d'alerte, tout comme l'intensité médiatique aujourd'hui possible par l'usage des réseaux numériques lui offrent un nouveau visage, et en font un phénomène communicationnel à part entière.

\section{Alerte et lanceurs d'alerte, un intérêt scientifique récent}

C'est en Amérique du Nord que les premiers champs de recherche se sont ouverts sur l'alerte et les lanceurs d'alerte, en sciences de gestion (ressources humaines, management, finances et comptabilité), en sociologie mais également en sciences politiques et en droit. La question est complexe, et a été appréhendée à divers niveaux : individuel, organisationnel, public. En 
Europe, ce sujet est resté relativement peu traité jusqu'à la fin des années 2000, à l'exception de quelques recherches pionnières (Bernstein, Jasper, 1996 : Chateauraynaud, Torny, 1999).

Les recherches sur l'alerte sont donc originellement connotées et marquées par leur origine américaine, le whistleblowing, qui signifie littéralement « souffler dans le sifflet». Aux États-Unis, une première loi date de 1935 : National Labour Relations Act. En 1986, le False Claim Act permet à toute personne de dénoncer une fraude au niveau fédéral. En 1994, le Whistleblower's Protection Act concerne uniquement les employés du secteur public, des sociétés publiques et de la Veterans Administration. Mais la principale loi nordaméricaine, la loi Sarbanes-Oxley (SOX), 2002, a eu un impact international, entraînant dans de nombreux pays l'obligation de légiférer également. Elle prévoit que toute personne empêchant l'alerte encourt une peine de prison et une amende de 250000 dollars. Selon Françoise de Bry (2008), « l'approche américaine s'inscrit dans un contexte historique [...] fondé sur un contrat individuel entre le citoyen et l'État qui l'incite à protéger et contrôle les agissements contraires à l'intérêt collectif. »

En France, l'alerte a tardivement été reconnue dans le droit français, et elle « reste ponctuelle et d'introduction relativement récente ». La loi française contre la fraude fiscale et la grande délinquance économique et financière du 6 décembre 2013, stipule que les individus qui témoigneront de faits constitutifs d'un crime ou d'un délit seront donc protégés, dans le secteur privé comme dans le secteur public. «L'alerte éthique » est ainsi reconnue par le pouvoir législatif et elle permet de révéler des actes illicites ou dangereux pour autrui, touchant à l'intérêt général (Chareire Petit, Surply, 2008).

Le lanceur d'alerte n'a pas un statut égal des deux côtés de l'Atlantique. Le contexte d'énonciation n'a pas la même force sociale. Aux États-Unis par exemple, le magazine Times a élu "personnalités de l'année 2002 » trois femmes whistleblowers. Des films rendent hommage à ces héros ordinaires. En Europe, et plus précisément en France, ni l'heure ni les us n'en sont encore à favoriser ce qui peut être vu comme une délation.

Malgré ces différences, de nombreux traits sont communs de part et d'autre de l'Atlantique. En premier lieu, l'alerte peut être appréhendée comme « une situation asymétrique donnant lieu à des définitions concurrentes, où chaque partie en présence tente d'imposer sa perspective et de légitimer ainsi à la fois les pratiques qu'elle recouvre et celles qui en découlent »(Scher, 2008). La question de l'alerte et celle de son lanceur d'alerte posent immédiatement la question du respect des normes. Le contexte d'énonciation de l'alerte et le parcours de l'émetteur dans ses différents rôles et statut sont fondamentaux.

\section{Qui lance l'alerte ? Polymorphie des profils, mais changement de statut partagé}

Selon les études conduites par Near et Miceli (1996, 2004), il n'y a pas de trait particulier psychologique propre aux lanceurs d'alerte. Pas de propension 
marquée, ni de catégorie socio-professionnelle privilégiée. Il y a en revanche un trait processuel propre à la majorité des lanceurs d'alerte dans leur choix de communiquer l'alerte : ils adressent dans un premier temps systématiquement leur rapport en interne. C'est parce qu'ils ne trouvent pas de réponse adéquate à leur révélation qu'ils vont alors s'adresser aux médias, brisant ainsi routine et parfois omerta, et bravant des représailles d'autant plus violentes qu'ils doivent désormais assumer ce qui peut être perçu comme un délit d'initié, dénoncer de l'intérieur.

Un autre trait est également avancé pour la majorité d'entre eux, et qui tient au socle de leur prise de parole : ils agissent pour un intérêt supérieur, au nom de l'intérêt général et du bien commun, au nom du devoir et de la vérité (Glazer \& Glazer, 1989, Alford, 2001). Ils feront d'ailleurs valoir la dimension désintéressée de leur action (Bernstein, Jasper, 1996), l'aspect public, moral et éthique de leur dénonciation (Brodeur, Jobard, 2005). Ils opposent ainsi la vision du traître qui va leur être adjointe à celle de la vigie, du citoyen vertueux (Scher, 2008), recadrant leur prise de parole sous l'angle de la responsabilité, soutenue par une loyauté à des principes supérieurs. Selon le soutien du public, ils endosseront ainsi le rôle du martyr, de la croisade solitaire ou du héros (Akerström, 1991).

Ce positionnement connote fortement l'alerte, dès son émission, et le rôle pris par l'émetteur va cristalliser par la suite la poursuite de l'alerte, qui, inéluctablement, tend la majorité des cas vers sa diffusion dans l'espace public.

\section{«Souffler dans les médias " par défaut?}

Pour le lanceur d'alerte, le choix des médias est souvent le seul restant pour porter l'alerte. La voie juridique est souvent beaucoup trop longue et le temps de l'alerte, qui peut parfois correspondre à un temps de crise, est beaucoup plus court. La voie interne est devenue caduque. Il reste la voie médiatique qui permet l'interpellation publique des premiers contactés (l'organisation, qui se doit alors de réagir), mais également du public qui s'interpose comme juge et soutien dans la partie qui s'annonce.

Dans cette logique d'accession aux médias, les lanceurs d'alerte recherchent le relais de l'opinion publique. Le public constitue une pression normative conséquente, à la fois sur les médias et sur les acteurs. L'appréhension d'une sympathie publique a pour effet immédiat d'infléchir les décisions politiques.

Ils cherchent également la rupture qui bousculera suffisamment l'organisation pour que les alertes soient prises en considération. Pour avoir accès aux médias, outre qu'ils apportent le scoop, les lanceurs d'alerte vont alors adopter le statut de définisseur primaire. C'est bien ici qu'est l'enjeu médiatique (Callahan, Dworkin, 1994). Lorsque l'alerte passe, elle est qualifiée de "nouveauté », de "spectaculaire », d'« événement », et a toutes les chances de provoquer la rupture de réalité voulue par le lanceur. 


\section{De l'alerte à la controverse : le difficile passage de l'acte interne au problème public}

Pour le lanceur d'alerte, il s'agit donc souvent de transformer un acte interne en problème public, dans l'espace public, tel que théorisé par Habermas (1992). Les travaux sur les lanceurs d'alerte (Chateauraynaud, Torny, 1999) ont ainsi montré un continuum allant du cas de l'alerte « reconfinée » à celui de « crise politique »: une alerte crée un évènement médiatique en faisant proliférer les interventions, les prises de parole, les commentaires, les analyses.

L'accès aux médias n'est pourtant pas forcément acquis d'emblée. De nombreux auteurs ont analysé précisément certaines alertes, comme celle des éthers de glycol, celle de l'amiante (Jouzel, 2008), ou encore celle de l'usine d'incinération de Gilly-sur-Ysère (Arpin 2010) et sa pollution à la dioxyne, et ont clairement essayé de comprendre ces silences médiatiques. Malgré l'usage de plus en plus courant des réseaux sociaux dans les dernières alertes, l'accès aux médias d'information classique reste prépondérant dans la poursuite de l'alerte vers l'espace public.

Ici, quatre dynamiques interagissent, et peuvent, pour chacune d'entre elles prise séparément ou de façon combinée, faire taire l'alerte : le lanceur d'alerte en tant qu'émetteur, le journaliste, au double statut de récepteur et de grand émetteur, l'alerte en tant que message à décoder et encoder, et la force normative souvent personnalisée par les pouvoirs publics. Nous développons ces quatre forces l'une après l'autre pour plus de clarté, mais dans les faits, nous relevons des effets de seuil, de combinaison qui ouvrent de nouvelles perspectives de recherche : les dernières alertes (boues rouges, bisphénol, phtalates, PCB) semblent plutôt relever un "effet cocktail » ${ }^{2}$ : 'est la combinaison de fragilités (doutes sur l'émetteur, relative incompréhension du journaliste, message en dissonance par rapport à la norme...) qui semble bloquer l'alerte.

\section{La question de la crédibilité de l'émetteur}

Le lanceur d'alerte n'agit pas au sein d'une institution mais en tant que simple citoyen, que souvent rien ne distingue d'autres salariés si ce n'est la détention d'informations incitant à la rupture ou pouvant entraîner une crise.

La parole du lanceur d'alerte vient « en contre » : contre la norme, contre la routine, contre le "wrongdoing ». Le statut accordé à sa parole, la crédibilité et la légitimité de son propos peuvent porter toute la controverse à venir. Or, face à l'individu, le journaliste a plusieurs réactions possibles, qui vont du crédit immédiat à la non-considération de l'information.

Le lanceur d'alerte peut être considéré comme défaillant par le journaliste, qui souvent va enquêter à charge et à décharge : cette défaillance peut être l'objet de la non-publication de l'alerte. Le lanceur d'alerte prend le statut de

2 Nous reprenons ici une expression portée par les lanceurs d'alerte sur l'effet des produits chimiques, car elle est particulièrement pertinente pour qualifier cette combinaison de forces/dynamiques de diffusion vue par le prisme des Sciences de l'information et de la communication. 
source d'information, et dans le débat qui s'engage, l'enjeu va être de rester définisseur primaire, voire " expert ». La capacité à rester définisseur primaire est essentiellement donnée aux lanceurs d'alerte disposant d'une expertise scientifique, car il leur faut être capables de dégager « les points aveugles des dispositifs d'expertise instrumentés et routinisés " (Chateauraynaud, Hélou, Lemieux, Torny, 1997).

\section{Le journaliste, pivot de l'accession à l'espace public ?}

Le journaliste fonctionne également selon une logique d'évènement, de médiatisation face à un contexte. La pratique du journalisme va entrer en conflit avec le caractère officieux de la rumeur. Cette considération est à croiser avec la notion d'affaires et de non-affaires (Boltanski et al, 2007), laissée au libre arbitre du journaliste ou faisant l'objet d'un traitement judiciaire. Le journaliste est alors perçu comme un agent de dévoilement (Simmel, 1999).

L'une des principales difficultés pour le journaliste est l'accès à des preuves tangibles, qui corroborent celle du lanceur d'alerte. Pour être recevable et exploitable par le journaliste, le fait relevé, l'alerte, le rapport, doivent être « équipés » (Latour, Woolgar, 2005), ce qui va lui permettre de différencier la rumeur de l'affaire. Ici intervient la notion d'« embarras », telle qu'exprimée par Goffman (1991) : elle désigne les situations de défaillance ordinaire de nos cadres d'appréciation de la réalité.

Le rôle du journaliste est également de poser la question du contrôle, tel que défini par Thompson (2005), selon une trilogie de questionnements simples : ceux qui ont commis les actes incriminés (les fautifs), ceux qui les ont mis en accusation (les accusateurs), et ceux qui auraient dû les surveiller (les superviseurs). Ce questionnement conduit d'emblée à une logique de communication de crise et de gestion de la parole.

Enfin, les médias peuvent être amenés à reprendre l'alerte à leur compte, non seulement pour relancer le débat, mais pour ouvrir de nouveaux espaces de controverse proches. C'est ainsi que de nombreux documentaires ont été diffusés cette année sur les chaînes publiques, allant au-delà d'alertes lancées, révélant un travail d'investigation et élargissant l'espace de controverse et des prises de risques (Le Cauchemar de Darwin, Notre poison quotidien, etc.)

\section{L'alerte, entre rupture et diffusion}

Concernant l'alerte, sa chance de diffusion doit également tenir compte du contexte, de l'air du temps. La notion d'agenda setting (Mc Comb, Shaw, 1972) est à prendre en compte, ainsi que la valeur attachée à l'information, la "newsworthyness» (Tuchman, 1972).

L'alerte ici, présente de nombreux traits propres aux événements : c'est une rupture d'intelligibilité, c'est une occurrence, un surgissement dont l'impact va être sociologique, déboussolant les cadres interprétatifs. L'alerte est donc rupture, chaos, elle contient la même capacité de désordre que l'événement ; 
elle bouscule le contexte établi, la routine, l'organisation acceptée. Elle présente ce potentiel de saillance propre aux événements (Dearing, Rogers, 1988).

La question de l'accès aux médias et de leur dynamique relève donc de la sociologie des médias et, pour comprendre certains silences médiatiques, Stéphane Arpin (2010) fait appel à la difficulté de compréhension de la grammaire locale dans laquelle peut s'exprimer l'alerte, à l'impossibilité pour le journaliste de trouver des " prises critiques ».

\section{La prégnance de l'appareil normatif institutionnel}

Face au lanceur d'alerte, face au journaliste, un autre locuteur, souvent mis en cause par l'alerte, pèse de tout son poids dans l'imposition de la norme : l'État et ses institutions. Il s'agit bel et bien de briser alors toute la dimension normative contre laquelle l'alerte se porte en faux : or c'est souvent une norme tacite autour du non-dit, particulièrement difficile à déplacer. Selon Jean Jouzel (2008) « en se publicisant, l'alerte rompt ainsi la trame ordinaire de la confiance qui permet aux appareils qui gèrent le risque de soustraire leurs décisions au débat public».

Seul le travail d'enquête peut véritablement donner la parole aux silencieux. Il peut alors y avoir la restitution ex-post des points de vue, et parfois, la recréation d'une affaire (Lemieux, 2000).

\section{De l'usage croissant de l'alerte : un espace public réceptif, attentif et actif}

Le contexte dans lequel évoluent les lanceurs d'alerte a considérablement changé ces dernières années. Il est clair désormais que le schéma de communication dans lequel s'exprime l'alerte est complexe mais également particulièrement ouvert, notamment à la société civile.

\section{L'alerte, un bouclier dans un contexte de risque et d'insécurité ?}

Les notions de risque et d'insécurité sont fondamentales pour comprendre sur quelles bases théoriques s'appuie la réception des alertes. L'alerte est souvent associée à des risques sociaux, financiers, économiques, naturels, humains. Une fois diffusée par les médias, l'alerte prend la dimension de problème public dans la majorité des cas, et rejoint ainsi la catégorisation de risques potentiels qui déclenche l'entrée en jeu de nouveaux acteurs, experts, décisionnaires, mais aussi profanes.

La sélection et la catégorisation de ces risques s'expliquent de trois façons différentes : soit comme le résultat d'arbitrages opérés par les autorités publiques, soit comme le résultat de confrontations entre "société civile » et autorités publiques, soit comme le résultat de la manière dont de multiples acteurs définissent et construisent les problèmes.

Les recherches et différents terrains analysés (Pascual Espuny, 2007, 2010), nous incitent à privilégier comme principal cadre d'expression des 
alertes actuelles la deuxième option, celle de confrontations de plus en plus fréquentes entre la société civile et les autorités publiques en tant qu'instances normatives. Il existe souvent un décalage entre la gravité des risques et la perception de ceux-ci par l'opinion publique pour de très nombreuses raisons qui peuvent être d'ordre psychométrique par exemple, psychosociologique ou culturelle (Douglas, Widalsky, 1983, Douglas, 1985), ou qui croisent ces dimensions. Un rapport de forces de plus en plus important entre la société civile et ses représentants permet l'émergence de problématiques d'ordre public, les inscrit dans l'agenda politique et médiatique, et les engage dans une procédure d'exposition au risque. Callon et Rabeharisoa (1999) ont ainsi mis en perspective l'action de ces acteurs de la société civile, qui peuvent intervenir dans les procédures, qui croisent, collectent des données, voire peuvent solliciter des expertises. Nous rajoutons qu'ils se sont aujourd'hui également constitués en collectif de protection pour les lanceurs d'alerte (exemple : Réseau Santé Environnement), dans une "société du risque » (Beck, 1992) fortement médiatisée.

\section{Des controverses qui s'ouvrent aux savoirs profanes}

Ainsi, «face à l'incertitude, la hiérarchie paraît s'estomper entre savoirs savants et savoirs profanes. Face aux grands problèmes de société, l'expertise des acteurs de la société civile peut sembler valoir celle des experts scientifiques, des responsables politiques, etc. » (Roqueplo, 1997).

Cet état de fait est essentiellement rencontré en situation d'incertitude et de limites des savoirs scientifiques. C'est par exemple le cas autour de la question des nanotechnologies. Selon Callon, Lascoumes et Barthe (2001), on sort de l'espace gestionnaire délimité par la procédure d'exposition au risque pour entrer dans l'univers des controverses scientifiques, des débats publics, des forums hybrides. Cet état de fait est également possible grâce à la promptitude des nouveaux médias sociaux, à l'émergence d'une nouvelle citoyenneté, à des nouvelles pratiques (Hermite, 1996, Brown \& MastersonAllen, 1994, Lagadec, 1991).

Alors que les citoyens sont de mieux en mieux informés, de plus en plus organisés et plus aptes à s'exprimer via notamment les réseaux sociaux, Giddens (1990) souligne l'existence d'un décalage croissant entre d'un côté le monde vécu et familier et de l'autre des sous-systèmes institutionnels autonomisés et spécialisés, difficilement capables de gérer cette incertitude et les crises qui en découlent. L'importance des médias est alors essentielle, notamment dans la construction de scénarios riches de sens et d'émotion qui font défaut aux institutions dans la description des crises (Coombs, Holladay, 2007, Seon-Kyoung, 2009). 


\section{La société civile, récepteur avisé et nouvel émetteur de poids}

Enfin, l'évolution très récente des différentes crises montre une société civile qui se structure, où l'on constate que la parole du profane peut avoir autant d'impact que celle de l'expert (Lascoumes, 2002). Au niveau de l'espace public dans lequel se développe la controverse, la notion de leader d'opinion, développée par Katz en 1957 reprend toute sa place. Les phénomènes de « montée en généralité » posés par les médias et repris dans la société civile sont alors fondamentaux.

Si l'alerte permet la controverse, alors celle-ci devient un lieu de productivité sociale selon Pierre Lascoumes (2002), elle « ouvre la voie à la mise en politique » en même temps qu'à la " mise en recherche ». La controverse "enclenche un processus d'expérimentation à la fois scientifique et politique », où de nouvelles incertitudes seront mises à jour, où de nouveaux acteurs entreront en scène.

Comme Pierre Lascoumes, nous envisageons la controverse comme un processus d'apprentissage, qui permet la stabilisation d'un enjeu et « un nouveau régime d'énonciation de l'intérêt général ». «Si une controverse bien développée ne tend pas forcément vers le consensus, elle débouche sur un accord plus ou moins durable entre des majoritaires, accord soutenu de fait par le renoncement des minoritaires à déstabiliser la coalition précédente ».

Nous pensons que si l'alerte déclenche la controverse, alors celle-ci rendra publiques et visibles les incertitudes, les « wrongdoings ", et pourra à long voire à moyen terme réussir à changer des comportements.

\section{Conclusion}

Nous tenons à souligner les champs fertiles d'une recherche qui s'ouvre et qui a besoin, de notre point de vue, de compétences transversales, au carrefour de la communication de crise, de la psychosociologie, des sciences politiques, des sciences de gestion.

Nous pensons par exemple que les crises alimentaires récentes sont de nouveaux cas de cette dynamique. Elles conduisent à des crises latentes et sont prises en charge par un réseau de lanceurs d'alerte sur la thématique de la santé et l'environnement.

Les domaines de recherche abondent, qui peuvent faire l'objet d'études empiriques qui valideraient les hypothèses et le chemin d'accès théorique qui ont fait l'objet de cet article exploratoire.

\section{BIBLIOGRAPHIE}

AKERSTRÖM M, Betrayal and betrayers: the sociology of treachery, New Brunswick, Transaction Publishers, 1991.

ALFORD C. F, Whistleblowers: Broken Lives and organizational Power, Cornell University Press, 2001. 


\section{C\&O n45}

APPELBAUM S. H, GREWAL K., MOUSSEAU H., « Whistleblowing: international implications and critical case Incidents ", Journal of American Academy of Business, Cambridge, Sept. 2006, 10, 1.

ARPIN S., « Pourquoi les médias n'en parlent-ils pas ? " Réseaux n 159, 222, 2010.

BECK U., Risk society: Towards a new Modernity, Sage Publications, London, 1992.

BERNSTEIN M, JASPERJ. M, «Les tireurs d'alarme dans les conflits technologiques. Entre intérêt et crédibilité », Politix, n 79, 2008.

BOURNOIS F, BOURION C, «Les nouveaux gardiens de l'éthique, ou la vigilance citoyenne ", Revue internationale de psychosociologie, 2008/3, vol. 14, 2008.

BOLTANSKI L., CLAVERIE E., OFFENSTADT N. Van DAMME S., Affaires, scandales, grandes causes. De Socrate à Pinochet, Paris, Stock, 2007.

BRODEUR J.-P., JOBARD F., Citoyens et délateurs, Paris, Autrement, 2005.

BROWN P. MASTERSON-ALLEN, "The toxic waste movement: a new type of activism ", Society and Natural resources, vol. 7, n 3, 1994.

CALLAHAN E. S, DWORKIN T., "Who blows the whistle to the media, and why: organizational characteristics of media whistleblowers ", American Business Law Journal, vol. 32, 1994.

CALLON M., "Pour une sociologie des controverses technologiques », Fundamentae Scientae, vol. 2, n 3/4, 1981, 381-399.

CALLON M., LASCOUMES P., BARTHE Y., Agir dans un monde incertain, Paris, Seuil, 2001.

CALLON M., RABEHARISOA V., Le pouvoir des malades : l'association française contre les myopathies et la recherche, Paris, Presse de l'École des Mines, 1999.

CHAMPAGNE P., "La manifestation. La production de l'évènement politique », Actes de la recherche en sciences sociales, vol. 52, 19-41, 1984.

CHAREIRE PETIT, SURPLY J., « Du whistleblowing à l'américaine à l'alerte éthique à la française : enjeux et perspectives pour le gouvernement d'entreprise ", M@nagement, vol. 11, n² 2, 2008.

CHATEAURAYNAUD F., TORNY D., Les sombres précurseurs. Une sociologie pragmatique de l'alerte et du risque, Paris, éd. EHESS, 1999.

COOMBS W. T., HOLLADAY S. J, « The negative communication dynamic: exploring the impact of stakeholder affect on behavioural intentions ", Journal of Communication Management, 2007.

DEARING J., ROGERS E., Agenda setting, SAGE Publications, 1996.

DE BRY F., "Salariés, héros ou délateurs ? Du whistleblowing à l'alerte éthique ", Lettre du management responsable, 2006.

DOUGLAS M., WIDALSKY A., Risk Culture. An essay on the selection of technological and environmental dangers, Berkeley, University of California Press, 1983.

DOUGLAS M., Risk acceptability according to the social sciences, London, Routledge \& Kegan Paul, 1985.

FISHER J. et al., Whistleblowing on the web, St Louis University, juin, www.be.edu, 1999. 
GIDDENS A., The consequences of modernity, Cambridge University Press, Cambridge, 1990.

GOFFMAN, La Présentation de soi, Éditions de Minuit, coll. « Le Sens Commun », 1973.

GLAZER M., GLAZER P., The whistleblowers: exposing the corruption in government and industry, New York, Basic Books, 1989.

HABERMAS J., L'espace public, Paris, Payot, 1992.

HERMITTE M.A., Le sang et le droit. Essai sur la transfusion sanguine, Paris, Le Seuil, 1996.

JOUZEL J. N., « La dénonciation du problème des éthers de glycol en France. Les organisations syndicales face à l'après-crise de l'amiante ", Revue française des affaires sociales, 2008/2, 2008.

KATZ E., "The Two-Step Flow of Communication: An Up-To-Date Report on an Hypothesis”, Public Opinion Quaterly (1957) 21 (1): 61-78.

KECK F., "Conflits d'experts. Les zoonoses, entre santé animale et santé humaine », Ethnologie française n ${ }^{\circ}$ 39, 2009.

LAGADEC P., La gestion des risques, Mc Graw Hill, 1991.

LATOUR B, WOOLGAR S., Laboratory Life: The Social Construction of Scientific Facts, Beverly Hills, Sage Publications, 1979 ; rééd. Princeton, Princeton University Press, 1986.

LASCOUMES P., "De l'utilité des controverses sociotechniques ", Journal international de la bioéthique 2002/2, vol. 13, 68-79, 2002.

LEMIEUX C., Mauvaise presse. Une sociologie compréhensive du travail journalistique et de ses contraintes, Paris, Métaillié, 2000.

MARTENS L., KELLEHER A., "A global perspective on whistleblowing», International Business Ethics review, vol. 7, issue 2, 2004.

MC COMB M., SHAW D., "The agenda setting function of mass media», Public Opinion Quaterly, vol. 36, 1972.

NEAR J. P, MICELI M. P., «Whistleblowing: Myth and reality», Journal of Management, 22-3, 502-527, 1996.

NEAR J. P., REGH M.T, VAN SCOTTER J. R., MICELI M. P., «Does wrongdoing affect the whistleblowing process?» Business Ethics Quaterly, 14:2, 2004.

PASCUAL ESPUNY C., "Controverses, autour de Reach », La Revue Internationale de Psychosociologie, 38, Paris, 2010.

PASCUAL ESPUNY C., " Le développement durable, promesse d'un changement paradigmatique ? Étude d'un processus discursif et négocié. Un exemple : Reach », Thèse en sciences de l'information et de la communication, Paris 4 Sorbonne, 2007.

QUERE L., "Les dispositifs de confiance dans l'espace public », Réseaux, vol. 23, $\mathrm{n}^{\circ} 132,185-217,2005$.

ROQUEPLO P., Entre savoir et décision, l'expertise scientifique, Versailles Cedex, Éditions Quæ « Sciences en questions », 1997.

SCHER S, « L'alerte comme forme de déviance : les lanceurs d'alerte entre dénonciation et trahison », Déviance et société, vol. 32, n², 2008. 
SCHUDSON M., «Was there ever a public sphere», in C. Calhoun, ed., Habermas and the public sphere, Cambridge, Mass, MIT Press, 1993.

SEON-KYOUNG A., GOWER K. K., «How do the media frame crises? A content analysis of crisis new coverage», Public relations review, vol. 55, 2009.

SIMMEL G., Sociologie. Étude sur les formes de la socialisation, Paris, Presses universitaires de France, 1999.

THOMPSON D. F., Restoring responsibility, Cambridge University Press, 2005.

TUCHMAN G., Making Nerws. A study in the construction of reality, New York, The Free Press, 1972.

Résumé : Les dernières crises concernant notamment la santé environnementale montrent un nouveau visage de la société civile qui, non seulement s'approprie les sujets les plus techniques et spécialisés, mais devient lanceur d'alerte. Quel est ce nouveau visage de la société civile? Quel nouvel espace public se dessine ? Nous proposons une relecture communicationnelle des controverses et de la notion d'expert et nous envisageons les notions de crise et de risque, au regard de la portée communicationnelle particulièrement performante des lanceurs d'alerte.

Mots-clé : communication, alerte, espace public, risque, expertise.

Abstract : The recent crises including environmental health show a new face of civil society, which not only appropriates the more technical and specialized topics, but becomes whistleblower. What is the new face of civil society? What new public space emerges? We propose a communicational replay of the controversy and the notion of expert and we are considering the concepts of crisis and risk, with regard to the particularly powerful communicative scope of alerts launchers.

Keywords: communication, alert, public space, risk, expertise. 